\title{
Aerosol-based detectors for liquid chromatography: do they have a role in bioanalysis?
}

\author{
"With improved sample preparation methods and more efficient chromatographic separations, it should be possible to apply \\ aerosol-based detection to somewhat more challenging biological samples."
}

Analysis of the components of biological samples can be extremely challenging due to low concentrations of the compounds of interest, high concentrations of other compounds and the complexity of samples. In order to obtain quality measures of the concentrations of the analytes of interest, instrument-based methods must show good selectivity, high sensitivity and the ability to calibrate the instrument for the analytes of interest. Sample preparation steps and HPLC separations provide some of the selectivity needed.

"The earliest aerosol-based detectors evaporative light scattering (ELS) detectors were first described in the 1960s but were further developed in the late 1970s and early 1980s to meet the need for universal detection."

Before LC-MS instruments became readily available, analysts often faced a choice of using UV detection or a more sensitive and selective alternative, such as fluorescence or electrochemical detectors. This choice would depend on whether the analysis was aimed more at specific compounds or comprehensive analysis. For many compounds, especially compounds without chromophores, conversion of the compounds to fluorescent or UV-absorbing derivatives was an additional step, adding to sample-preparation complexity but resulting in greater selectivity, with only a fraction of the compounds eluting from the column being detected. The increasing availability and performance of LC-MS instruments has changed the focus of many analyses; LC-MS gives excellent sensitivity and selectivity and responds to a wide range of analytes, it has therefore become increasingly important in bioanalysis. The main shortcomings of LC-MS are the higher equipment and operation costs, difficulties in ionizing some analytes and variability in response (which makes the quantitative determination of analytes without standards difficult). Variability in response is one limitation that can be overcome through the use of a more universal-type detector.

\section{Aerosol-based detectors}

In the early period of HPLC development, one apparent limitation was the lack of a detector that was economical, sensitive and universal in the way that the flame ionization detector (FID) is for GC. Refractive index detectors have been used as universal detectors, although they have limited sensitivity and are not compatible with gradient elutions. UV detectors are also used as universal detectors, despite the wide range of sensitivities for compounds, including compounds without chromophores. The earliest aerosolbased $(\mathrm{AB})$ detectors - evaporative light scattering (ELS) detectors - were first described in the 1960s [1] but were further developed in the late 1970s and early 1980s to meet the need for universal detection [2-5]. While neither inexpensive nor highly sensitive compared with FIDs for GC, ELS detectors are universal for compounds of low volatility. These detectors have seen a gradual increase in sensitivity and usage since the early 1980s. Many of the early applications were aimed at compounds of biological origin with weak chromophores, such as lipids and carbohydrates [3,6]. More recent reviews of ELS detection have shown some analysis of biological samples including serum and urine, although more common samples are food and pharmaceuticals [7]. Numerous companies sell ELS detectors. More

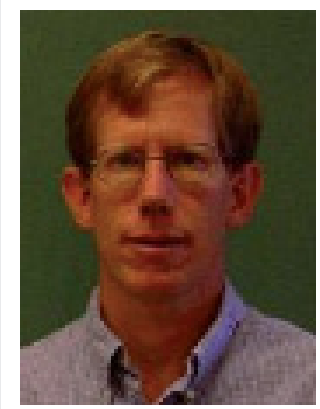

Roy W Dixon

California State University, Sacramento, 6000 J Street,

Sacramento, CA

95819-6057, USA

Tel.: + | 9162786893

Fax: + I 9162784986

Email: rdixon@csus.edu 
recently, two more advanced $A B$ detection methods have been developed, condensation nucleation light-scattering (CNLS) detection [8,9] and charged aerosol (CA) detection (originally termed aerosol charge detection) [10,11], which have greater sensitivity than ELS detection.

$A B$ detectors, a term adapted from Koropchak et al., all function by converting the effluent exiting a HPLC column into an aerosol and then detecting some property of the resultant aerosol with an 'aerosol detector' (Figures I \& 2) [12]. The conversion phase typically occurs through pneumatic spraying of the effluent into a stream of nitrogen, followed by evaporation of the spray droplets in an evaporation chamber. It is often said that $\mathrm{AB}$ detectors can respond to any compound that is less volatile than the solvent. However, it should be pointed out that response is best for 'nonvolatile' compounds and attention must be paid to ensure that nonvolatile additives or contaminants are not present in solvents. In ELS detectors, the aerosol property detected is light scattering. The general principle is that when higher concentrations of analyte are present, the resulting aerosol particles are larger and scatter more light. When concentrations are low (under $\sim 1 \mu \mathrm{g} \mathrm{ml}^{-1}$ ), residual particles are too small to scatter light efficienctly, thus limiting sensitivity [8].

In CNLS detection, sensitivity is greatly increased by 'growing' particles through condensation of a liquid (water or butanol) to sizes where detection by light scattering becomes efficient $[8,9,12]$. The aerosol detectors used in CNLS detection are used for clean rooms where particles in the order of nanometers can be detected at concentrations as low as one particle per liter. This indicates that, in principle, an extremely sensitive detector is possible. In practice, sensitivity is often limited by solvent impurities (solvents of the lowest listed residue concentrations should always be

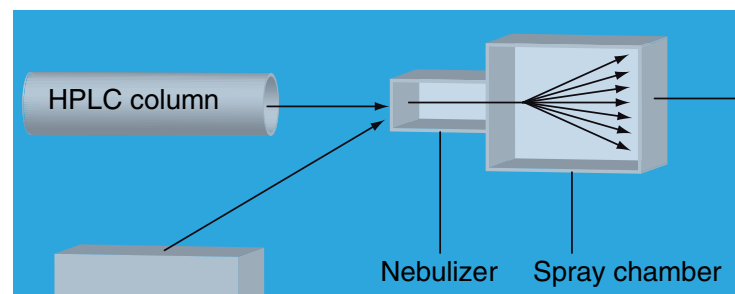

Nitrogen

Nebulizer Spray chamber

\section{Evaporation chamber}

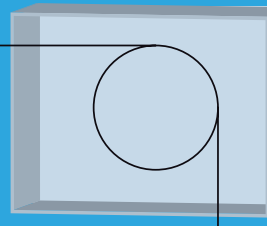

Aerosol detector
Bioanalysis @ Future Science Group (2009)

Figure 1. Generic aerosol-based detector. used) and column bleed [9]. CNLS detection was found to result in detection limits two orders of magnitude less than ELS detection [13]. Sensitive CNLS detection has been demonstrated in the analysis of sugars in urine [14], biogenic amines in fish samples [15] and lipids in egg yolk samples [16]. A commercial CNLS detector for HPLC has recently been produced [101].

\section{“Aerosol-based detectors ... all function by converting the effluent exiting a HPLC column into an aerosol and then detecting some property of the resultant aerosol with an 'aerosol detector."}

Small aerosol particles are also efficiently charged through diffusion of molecular ions to particles' surfaces; this is the basic principle behind smoke detectors. Although the exact charging mechanism is somewhat different in the first detector described [9] and the commercial instrument [10], in both cases a corona discharge supplies the molecular ions and the charged particle flux is measured by collecting charged particles on a particle filter electrically connected to an electrometer. While it is not possible to detect single charged particles, charging is most efficient for small particles, giving CA detectors sensitivity much higher than ELS detectors and comparable with CNLS detectors [9]. Sensitive CA detection has been observed for lipids [17] and sugars [18,19], as well as degradation products [20].

\section{"Concentrations of unknown compounds can be estimated when standards are not available or prohibitively expensive..."}

Both CNLS detection and CA detection have been applied to microbore columns, with results showing that mass detection limits can be reduced to picogram levels by using smaller bore columns [16,21]. Presumably, this is because conversion to an aerosol is more efficient at lower flow rates.

While all of the aforementioned $\mathrm{AB}$ detection methods described are universal, it is conceivable that future $A B$ detectors could use other aerosol properties that depend on aerosol components, thus yielding a selective $A B$ detector. Besides the $\mathrm{AB}$ detectors mentioned previously, other detectors, such as atomic spectrometers and LC mass spectrometers, also involve aerosol production, although only as a step in converting analytes to products detected in the gas phase. Other 


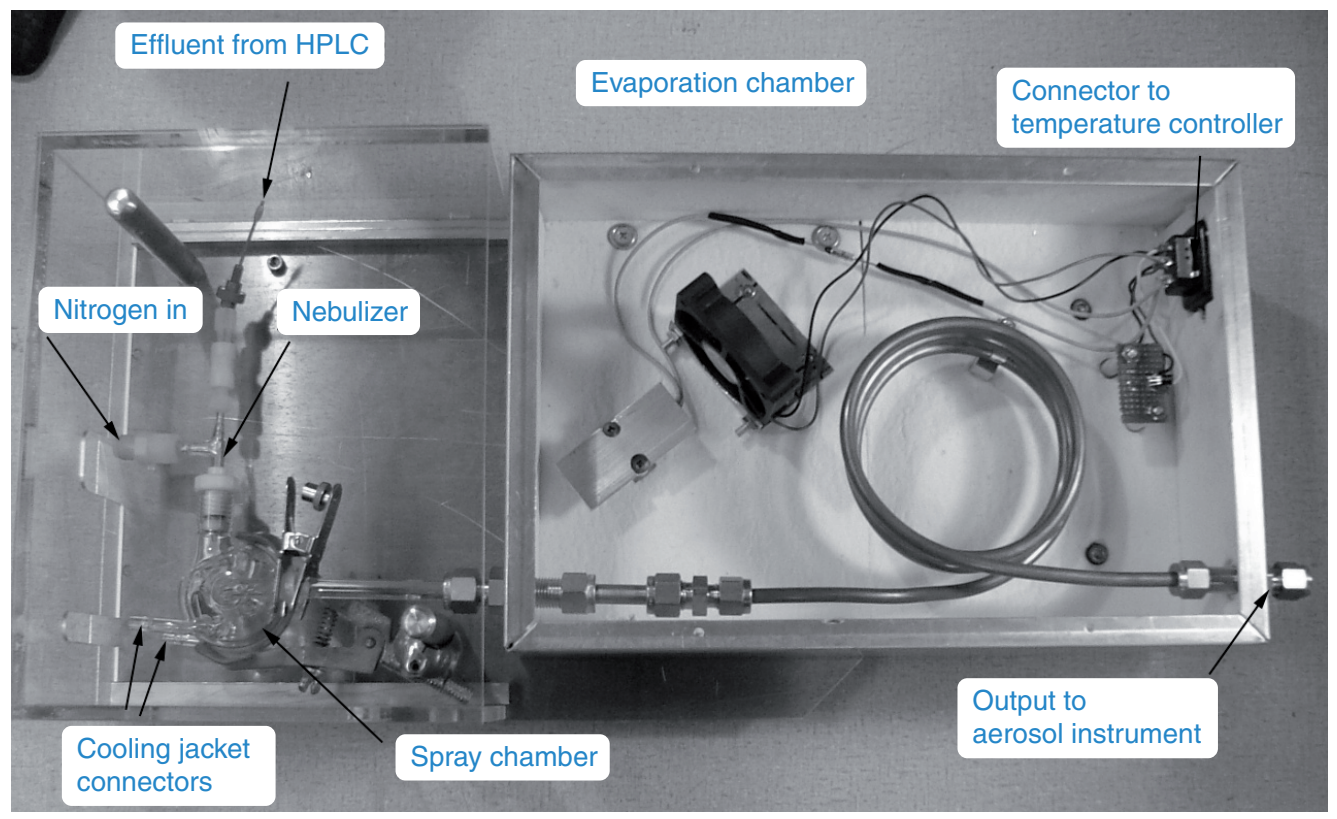

Figure 2. System used by the author for converting HPLC effluent to an aerosol.

detection strategies could use aerosol technology to separate aerosol particles from surrounding volatilized solvent, which could lead to a new type of FID.

\section{Application to biological samples}

In the application of $\mathrm{AB}$ detectors to biological samples, the goals of the project must be kept in mind. If the goal is to perform a comprehensive analysis of a compound class that makes up a significant fraction of the nonvolatile sample mass, $\mathrm{AB}$ detectors are practical alternatives to other methods. Since the AB response is not highly dependent on compound composition, concentrations of unknown compounds can be estimated when standards are not available or prohibitively expensive. This is commonly the case when examining metabolites or complex biological compounds such as glycosides or glycoprotein oligosaccharides. On the other hand, if the goal is to determine one to several compounds present at trace levels in complex samples, insufficient selectivity can limit the ability to meet the goal. This author and a student unsuccessfully attempted to apply a method developed for measuring the concentration of a sugar in atmospheric aerosols to urine samples [18]. In aerosol samples, the sugar was present at the percent levels, but, in urine, other components overwhelmed the analyte peak. While the sensitivity of CNLS detection and CA detection may be sufficient for trace analysis, the method selectivity must also be high. However, in cases where sample preparation methods can greatly reduce sample complexity (e.g., extraction of lipids from biological samples), trace level constituent analysis should be possible. Additionally, improvements in chromatographic resolution, including the move to smaller-packing particle sizes and advanced methods such as 2D chromatography may make a less selective detector more successful in the analysis of complex samples.

\section{Conclusion}

AB detection methods will work best for samples with relatively high fractions of analyte or with selective separation methods. However, with improved sample preparation methods and more efficient chromatographic separations, it should be possible to apply $\mathrm{AB}$ detection to somewhat more challenging biological samples. There is also an advantage when there are calibration challenges (e.g., no standards) that make selective MS detection difficult for quantification.

\section{Financial \& competing interests disclosure}

The author has previously consulted for Environmental Science Associates Inc. on charged aerosol detection. The author has no other relevant affliations or financial involvement with any organization or entity with a financial interest in or financial conflict with the subject matter or materials discussed in the manuscript. This includes employment, consultancies, honoraria, stock ownership or options, expert testimony, grants or patents received or pending, or royalties.

No writing assistance was utilized in the production of this manuscript. 


\section{Bibliography}

1 Ford DL, Kennard W. Vaporization analyzer. J. Oil Colour Chem. Assoc. 49, 299-313 (1966).

2 Charlesworth JM. Evaporative analyzer as a mass detector for liquid chromatography. Anal. Chem. 50, 1414-1420 (1978).

3 Macrae R, Dick J. Analysis of carbohydrates using the mass detector. J. Chromatogr. 210, 138-145 (1981).

4 Stolyhwo A, Colin H, Guiochon G. Use of light scattering as a detector principle in liquid chromatography. J. Chromatogr. 265, 1-18 (1983).

5 Mourey TH, Oppenheimer LE. Principles of operation of an evaporative light-scattering detector for liquid chromatography. Anal. Chem. 56, 2427-2434 (1984).

6 Stolyhwo A, Colin H, Guiochon G. Analysis of triglycerides in oils and fats by liquid chromatography with the laser light scattering detector. Anal. Chem. 57, 1342-1354 (1985).

7 Megoulas NC, Koupparis MA. Twenty years of evaporative light scattering detection. Crit. Rev. Anal. Chem. 35, 301-316 (2005).

8 Allen L, Koropchak JA. Condensation nucleation light scattering: a new approach to development of high-sensitivity, universal detectors for separations. Anal. Chem. 65, 841-844 (1993).

9 Allen L, Koropchak JA, Szostek B. Condensation nucleation light scattering detection for conventional reversed-phase liquid chromatography. Anal. Chem. 675, 659-666 (1995).

10 Dixon RW, Peterson DS. Development and testing of a detection method for liquid chromatography based on aerosol charging. Anal. Chem. 74, 2930-2937 (2002).

11 Gamache PH, McCarthy RS, Freeto SM et al. HPLC analysis of nonvolatile analytes using charged aerosol detection. LCGCN. Am. 23, 150-161 (2005).

12 Koropchak JA, Magnusson LE, Heybroek $M$ et al. Fundamental aspects of aerosol-based light-scattering detectors for separations. Adv. Chromatogr. 40, 275-314 (2000).

13 Koropchak JA, Heenan CL, Allen LB. Direct comparison of evaporative lightscattering and condensation nucleation light-scattering detection for liquid chromatography. J. Chromatogr. A 736, 11-19 (1996).

14 Wang Q, Sadain S, Koropchak JA. Investigation of the condensation nucleation light scattering detector (CNLSD) for carbohydrates with ion-exchange and hydrophilic interaction separations. Am. Biotechnol. Lab. 19, 22-24 (2001).

15 Sadain S, Koropchak JA. Condensation nucleation light scattering detection for biogenic amines separated by ion-exchange chromatography. J. Chromatogr. A 844, 111-118 (1999).

16 Yang X, Koropchak JA. Condensation nucleation light scattering detection with microbore liquid chromatography for lipid analysis. J. Microcolumn Sep. 12(4), 204-210 (2000).

17 Moreau RA. Analysis of lipids via HPLC with a charged aerosol detector. Lipids 41, 727-734 (2006).

18 Dixon RW, Baltzell G. Determination of levoglucosan in atmospheric aerosols using high performance liquid chromatography with aerosol charge detection. J. Chromatogr. A 1109, 214-221 (2006).

19 Inagaki S, Min JZ, Toyo'oka T. Direct detection method of oligosaccharides by high-performance liquid chromatography with charged aerosol detection. Biomed. Chromatgr. 21, 338-342 (2007).

20 Cascone A, Eerola S, Ritieni A, Rizzo A. Development of analytical procedures to study changes in the composition of meat phospholipids caused by induced oxidation. J. Chromatogr. A 1120, 211-220 (2006).

21 Hazotte A, Libong D, Matoga M, Chaminade P. Comparison of universal detectors for high-temperature micro liquid chromatography. J. Chromatogr. A 1170 , 52-61 (2007).

\section{Website}

101 Quant Technologies LLC. Manufacturing company that designs, manufactures, sells and services specialized scientific instrumentation to measure physical, chemical and biological properties of airborne particles www.quanttechnologies.com 